\title{
¿Dónde están las mujeres en la integración regional? Análisis y propuestas desde el MERCOSUR
}

\author{
Where are the women in regional integration? An insight from MERCOSUR
}

DOI: https://doi.org/10.22456/2178-8839. 113897

Leticia González

Universidad de Buenos Aires, Ciudad Autónoma de Buenos Aires, Argentina lgonzalezinfantino@gmail.com

Daniela Vanesa Perrotta CONICET/Universidad de Buenos Aires, CONICET/Universidad de Buenos Aires, Argentina danielaperrotta@gmail.com

\section{Resumen}

Este trabajo visibiliza las ausencias y muestra la riqueza del estudio desde prismas feministas para establecer una agenda de investigación integral de la integración regional en el MERCOSUR y que recupere el rol de las colectivas de mujeres y diversidades en la construcción de región a partir de su movilización, participación y reclamo. Se realiza un estudio de una selección de casos para identificar los movimientos contestación y participación de las mujeres en la construcción de región y la visibilizar las ausencias de la literatura disponible. Para ello, este trabajo se divide en tres secciones. La primera presenta un breve estado de la cuestión sobre la incorporación de los feminismos en el campo de estudios de la integración regional y el regionalismo. Desde esta perspectiva incorporamos a los feminismos decoloniales como una estrategia para el abordaje de la agenda invisible de las mujeres. La segunda sección presenta una descripción de cómo el MERCOSUR ha incorporado (o no) políticas de géneros y analiza dos casos puntuales (agricultura familiar y educación). A partir de lo anterior, la tercera sección aborda, a modo de conclusión, una propuesta para el desarrollo de una agenda de investigación.

Palabras claves: Feminismos; Enfoque descolonial; Integración regional:

\begin{abstract}
The objective of this work to make the absences visible and show the richness of the study from feminist lenses to establish a comprehensive research agenda of regional integration in MERCOSUR and that recovers the role of women's and diversities collectives in the construction of the region from of their mobilization, participation, and claim. A study of a selection of cases is carried out to identify the protest movements and participation of women in the construction of the region and to make visible the absence of the available literature. For this, this work is divided into three sections. The first presents a brief state of the art on the incorporation of feminisms in the field of studies of regional integration and regionalism. We incorporate decolonial feminisms as a strategy to address the invisible agenda of women. The second section presents a description of how MERCOSUR has incorporated (or not) gender policies and analyzes two specific cases (that of family farming and that of education). Based on the above, the third section addresses, by way of conclusion, a proposal for the development of a research agenda.
\end{abstract}

Keywords: Feminism; Decolonialism; Regional integration; 


\title{
Introducción
}

\begin{abstract}
"Fue una estrategia de la secretaría [de Agricultura Familiar de Argentina]: si venían varones, venía uno sólo. Si no, se le podía dar la oportunidad de que venga otro acompañante si era mujer o joven. Así empezamos y se empezó a cruzar el tema transversalidad de género que [la secretaría] lo tenía muy incorporado en su gestión. Entonces, nos allanó todo el camino [...]" (entrevista a representante de Organización de la Agricultura Familiar de Argentina, 2017)
\end{abstract}

"Nosotros los/as parlamentarios/as de las Delegaciones de Argentina, Paraguay y Uruguay [... declaramos] reivindicar los derechos de la identidad de género tomando las medidas necesarias para acabar con la discriminación [...]" (Declaración 2019 del ParlamentoJuvenil del MERCOSUR)

“-¿Por quéno invitan mujeres a ese panel [en centro universitario de referencia sobre in tegración] si hay muchas que podrían participar?

-Porque las mujeres [académicas] que trabajan integración no son generalistas, hacen agendas -como $X$ salud, vos educación... - entonces no tienen el big picture para un panel asi" (conversación de una de las autoras con un colega, 2016)

Este trabajo parte de vivenciar la invisibilización de las mujeres y diversidades en el estudio de los procesos de integración regional latinoamericana desdelas Relaciones Internacionales (RI), tanto en sus agendas de políticas públicas y cooperación regional como en el conocimiento académico producido desde posiciones subalternas (mujeres, indígenas o quien se aleje de los cánones consagrados de la ciencia). Por lo tanto, nuestro punto de partida es recuperar la pregunta sencilla pero sumamente potente del momento en que los enfoques feministasirrumpieron en la gramática androcéntrica de las RI a fines de los años ochenta: ¿dónde están las mujeres? (ENLOE, 2014). Desde entonces, los (diversos) feminismos empezaron a tejer puentes, posibilitando que más autoras y autores cuestionen sus agendas y metodologías de investigación, así como sus praxis. ¿Dónde están las mujeres en el MERCOSUR?, es la pregunta que nos interpela.

Como investigadoras de RI, nuestras reflexiones se han orientado a pensar las dinámicas construcción de la región desde miradas autóctonas, que se asientan en aquellos y aquellas que no tienen vozo, más bien, cuya voz no es escuchada ni su acción tomada en cuenta para comprender los procesos políticos. Y ello desde una posición que se revela como triplemente periférica: primero, estudiamos un tema periférico para las RI: procesos de integración regional; segundo, lo hacemos desde agendas que son consideradas poco gravitantes, ya que no abordamos ni cuestiones comerciales (economía política internacional) nijurídicas (derecho dela integración) y, tercero, somos investigadoras mujeres ylatinoamericanas. Esto es, como expresa la cita inicial que recuperamos textual, no estamos "autorizadas" a teorizar sobre "el” proceso de integración (ni siquiera cuando es nuestra región de pertenencia), sino que a comentar alguna cuestión menor conforme el tema que seguimos; $y$ mucho menos a poder generar conocimiento que discuta, interpele y cuestione el conocimiento estándar. Así, en esta disputa por visibilizar más integralmente procesos, actores y actoras de integración por fuera de las gramáticas canónicas, algunos temas quedan sin respuesta desdelas herramientas teóricas con l as que contamos.

En nuestras agendas de investigación, estos límites androcéntricos se hacen visibles. Para explicar la construcción de la agenda de agricultura familiar en el MERCOSUR, la participación de mujeres representado a las organizaciones del sector resulta un elemento central, que no ha sido debidamente considerado en las teorías que intentan explicar los procesos de integración regional. Las relaciones de género al interior de este sector social y productivo -un sector, por otra parte, sumamente masculinizado- y las estrategias de acción que se han propuesto en el seno de la reunión para trastocarlas son núcleos centrales para contribuir a la comprensión de su devenir y sus dinámicas. También lo son las formas de organización y funcionamiento propuestas dentro de la reunión desde los espacios de género (GONZÁLEZ; 2018). En el caso de la agenda educativa del MERCOSUR, sibien la temática que aborda es una sumamentefeminizada, no se incluyen espacios formales para el tratamiento de cuestiones sobre géneros y educación. Por un lado, no se han podido regionalizar avances normativos y de políticas que se desarrollaron en algunos países por la heterogeneidad de posiciones nacionales (el caso de las legislaciones sobre educación sexual integral es ilustr ativo de las divergencias). Por el otro, la 
obturación de instancias de participación formalizadas impide el avance de las discusiones y su procesamiento regional por la vía de consensos. Así, las propuestas que han quedado plasmadas en declaraciones regionales son las que han surgido en las ediciones del Parlamento Juvenil del MERCOSUR. Asimismo, la imposibilidad de participación de organizaciones sindicales y sociales generó un espacio regional menos permeable a las demandas desde los feminismos, porque los espacios institucionales nacionales en los que se discuten estas agendas al interior de los Ministerios nacionales se encuentran alejados de aquellos que participan en las instancias del MERCOSUR educativo (PERROTTA;2019).

Por lo expuesto, es el objetivo de este trabajo visibilizar las ausencias y mostrar la riqueza del estudio desde primas feministas para establecer una agenda de investigación integral de la integración regional en el MERCOSUR y que recupere lo que tienen en común los tres elementos señalados (que coinciden con las tres citas iniciales): el rol de las colectivas de mujeres y diversidades en la construcción de región a partir de su movilización, participación y reclamo. Consideramos que los feminismos pueden ofrecernos nuevos constructos y habilitar nuevas preguntas que hasta ahora no nos hemos hecho, nuevas respuestas que aún no hemos dado. Esto se justifica en que los movimientos de mujeres que han irrumpido con fuerza en las arenas públicas de todos los países de la región han traído nuevos cuestionamientos y visibilizando realidades calladas, han impactado en la política y en las políticas en múltiples agendasy niveles.

Desde estos múltiples lugares, urge la necesidad de reflexionar acerca de los vínculos entre las RI, la integración regional y los feminismos. No obstante, recorrer esta vinculación no implica relatar (ni contabilizar) dónde están las mujeres desde una perspectiva de la experiencia, sino ir más allá para plantear cómo estos enfoques nos pueden ayudar a comprender teóricamentelos procesos que buscamos explicar.

Desde una mirada asentada en los enfoques feministas descoloniales ${ }^{1} \mathrm{y}$ a partir de un recorrido descriptivo y exploratorio, en este artículo proponemos una forma de aproximarnos las diversas aristas de la integr ación regional en el MERCOSUR con nuevas lentes, que permitan comprender de forma más cabal cómo funcionan los procesos de integración en nuestra región, cuáles son sus avances, posibilidadesy desafíos. En otras palabras, a partir del estudio de una selec ción de casos (agendas de integración) del MERCOSUR, la identificación de los movimientos contestación y participación de las mujeres en la construcción de región y la visibilizacion de las ausencias de la literatura disponible, buscamos analizar cómo construir región desde prismas feministasy asíelaborar una agenda de investigación.

Para ello, este trabajo se divide en tres secciones. La primera presenta un breve estado de la cuestión sobre la incorporación de los feminismos en el campo de estudios de la integración regional y el regionalismo. La segunda sección presenta una descripción de cómo el MERCOSUR ha incorporado (o no) políticas de géneros y analiza dos casos puntuales (el de agricultura familiary el de educación). A partir de lo anterior, la tercera sección aborda, a modo de conclusión, una propuesta para el desarrollo de una agenda de investigación.

En términos metodológicos, se recurrió a la investigación documental y a entrevistas semiestructuradas realizadas a informantes clave respecto del abordaje de las temáticas de género en diferentes espacios del MERCOSUR Para el procesamiento de la información se utilizaron técnicas computacionales y software especializado (AtlasTiversión 9.0). Los datos obtenidos fueron analizados a partir de la interpretación fundamentada (STAKE; 1995) y su tratamiento consistió en el análisis de contenido y/o textual y análisis crítico del discurso (KORNBLIT; 2004). Parte del material analizado corresponde a investigaciones previas encaradas por las autoras.

\section{Feminismos, relaciones internacionales y el campo de estudios de la integración}

En esta sección trazamos un recorrido escueto sobre laincorporación de los feminismos en el campo de estudios de la integración y su articulación con las RI. Se busca contextualizar la ausencia/presencia de los feminismos en este

${ }^{1}$ Usamos el término descolonial siguiendo a Bidaseca (2018) y Grosfoguel y Mignolo (2008). 
campo -tanto en su red central como la red latinoamericana-y presentamos los aportes del feminismo descolonial como punto de partida para una agenda de investigación.

\section{El campo de estudios de la integración regionaly la incorporación de los feminismos}

El estudio de la integración regional (y del regionalismo, luego) puede abordarse a partir de la noción de campo, en tanto sistema autónomo de relaciones sociales de fuerza que está determinado por la producción y control de formas específicas de capital simbólico, que, según unas particulares reglas de juego, configura posiciones dominantes o dominadas, y en su estructuración se constituye la estructura social (BOURDIEU; 2003; 2009). El campo científico se articula en torno a la autoridad científica y el monopolio de la competencia científica es la base sobrela cual se cimienta la lucha entre las y los actores. La hegemonía del campo está dada en torno al paradigma que se erige como dominante que, como todo sistema o configuración de actores y posiciones, el balance de poder es permanentementeinestableya que hay tensiones en permanente movimiento. Así, al interior de un campo de conocimiento es posible considerar la existencia de variados paradigmas que se tornan hegemónicos para explicar el estado actual del fenómeno en cuestión-en este caso, la integración regional-. El campo de estudios de la integración tiene una presencia gravitante de las RI, pero no se agota en esta disciplina. Esta característica de inter-multi-transdisciplinariedad es clave para comprender desde su complejidad a la región; pero también para analizar las articulaciones de los ylas autores del campo con sus disciplinas “originarias”.

Al detener la mirada en la articulación de este campo con las RI, se constata que el estudio de la int egración (primero) y del regionalismo (luego) ha sido periférico salvo en tres momentos de su desarrollo donde este objeto de estudio ganó popularidad. Estos tres momentos de mayor visibilizacion son: su creación, entre mediados de la década de los años cincuenta y mediados de los sesenta; su reconfiguración, en los años noventa a partir de los debates globalización versus regionalización; su contestación, con el boom de la construcción de región en América del Sur, África y Asia durante el primer lustro del milenio actual (PERROTTA;2018). A suvez, cadamomento se puede vincular con lo que se conoce como tipos históricos u olas de construcción de región ensayadas a nivel mundial: 1) el proceso de integración de Europa Occidental en el período inmediatamente posterior a la II Guerra Mundial marca el inicio de las Teorías (europeas) de la integración (TEI); 2) la reconfiguración y el avance de la integración europea a nuevas áreas y niveles de regulación, que marca una maduración y ampliación de las TEI, por un lado, y la creación de múltiples y diversos acuerdos regionales en todo el globo y su vinculación con el proceso de globalización que estimula el surgimiento de lo que se conoce como las Teorías del Nuevo Regionalismo (TNR); 3) el momento de agitación conceptual del campo dado por el dinamismo de algunas regiones, junto con la menor gravitación del proyecto de Europa Occidental que habilita el diálogoy la búsqueda de acuerdos entre las diferentes comunidades epistémicas para generar una agenda de elabor ación de teoría y que incluye propuestas quevan desde promover un programa de investigación desde el regionalismo comparado hasta desarrollar una propuesta orientada en políticas sectoriales y la relación de la construcción de región con la gobernanza global ${ }^{2}$. Vale destacar que este último momento, además, coincide con una mayor apertura de las RI en diferentes sentidos: apertura geopolítica a incorporar a actores y actoras del "Sur global” -para visibilizar, adicionalmente, los aportes locales a una disciplina global - y apertura a otras gramáticas, en las que se inscriben los enfoques descoloniales y las críticas desde la idea de raza como dispositivo.

Al mismo tiempo, la noción de campo permite incorporar como elemento de análisis que los y las actoras (constructos, teorías y metodologías) que lo componen también están atravesados por sus posiciones en el marco de la configuración de redes centrales y redes periféricas de producción de conocimiento. Así, contamos con autores y autoras más o menos cercanos al core del campo, asícomo al core de las RI; impactando en que por cada momento de popularización

\footnotetext{
${ }^{2}$ El recorrido por estos momentos y la articulación entre redes puede verse en Perrotta, 2018. Aquí nos centramos en la articulación de las y los actores del campo con las RI y la presencia de feminismos.
} 
se generen corpus de conocimiento en redes centrales que luego migran a otras redes subordinadas, dándose diferentes lógicas de articulación (imposición, absorción, resignificación). A la vez, un conjunto de conocimientos queda en posiciones subalternas, periféricas o invisibilizadas; en el mejor de los casos con cierto reconocimientoy circulación local, pero que no se toma en cuenta "seriamente" (léase como conocimiento autorizado) en las redes centrales ${ }^{3}$. Este es el caso, por ejemplo, del pensamiento latinoamericano para la integración, donde es posible trazar un derrotero desde los movimientos de lucha por independencia a nuestros días (PAIKIN, PERROTTA Y PORCELLI; 2016) ${ }^{4}$. Con todo, esta mirada desde el campo permite comprender la gramática del campoy cómo se inscriben los feminismos, al no poder escindir las producciones académicas de sus contextos de producción y cómo éstos se interseccionan con la (re)producción de la vida cotidiana.

Pues bien, ¿cuándo ingresan los prismas feministas al estudio de la integración?, ¿qué enfocan a lo largo del tiempo? Es posible responder estos interrogantes a partir de cómo se insertan en las TEIy TNR estas discusione s, primero; y aquello que se configura por fuera de esas dos redes centrales de conocimiento, luego. Este rodeo nos permiteincorporar, como un último acápite, las propuestas desde los feminismos descoloniales latinoamericanos.

Para el corpus de las TEI, los primeros abordajes se centraron en dos ejes: por un lado, la mujeres e integración y, por el otro, género e integración. El primer eje buscó responder esquemáticamente dónde están las mujeres en el proceso de integración y en las TEI, concluyendo en la posición marginal de las mujeresy la posición dominante de los hombres en lugares, procesos e instituciones clave. Aquí, la categoría de género es reducida a mujer, que se ve como una categoría unificada y estable con intereses comunes. Desde el segundo eje, se arrojó luz en cómo el género moldea los roles socialmente construidos asignados a los hombresy las mujeres en la integración, colocando a los primeros en un lugar más ventajoso; cómo el poder del género refleja relaciones desiguales; cómo diferente s valores vinculados a masculinidades y feminidades moldean el proceso europeo. Para estos trabajos el poder es una relación entre diversas personas corporeizadas y no solo entre instituciones o entidades políticas, que no sololimita las oportunidades de las mujeres al dominarlas, sino que también empodera a los sujetos de género a través de su resistencia a la dominación (LOMBARDO; KANTOLA;2019).

Desde estos primeros estudios se constataron los sesgos masculinos de estas teorías: miran procesos abstractos, analizan actores (mayoritariamente hombres), no sopesan el impacto diferencial de las estructuras políticas y económicas sobre mujeres, hombres y “minorías”; pero también, destacan que los primeros estudios fueron sobre políticas para la mujer (y no sobre el proceso de integración en su totalidad) (KRONSELL, 2005). Siguiendo a Lombardoy Kantola (2019), la inclusión de enfoques analíticos y metodológicos de los feminismos (tales comola deconstrucción, la interseccionalidad yla post-deconstrucción) permite mejorar la capacidad analítica de las TEI frente a los actuales desafíos de desintegración a partir de: vincular la teoría y la práctica, abordar las dinámicas de poder entre diferentes personas y sus cuerpos e identificar las oposiciones y contrafuerzas discursivas e interseccionales, así comolos contextos afectivos en los que se desarrollan los actuales procesos de desintegración e integración.

Con todo, Locher y Prügl (2009) consideran que los enfoques de género permiten revelar la dimensión ocul ta de la integración europea en tantola UE -como autoridad política-reorganiza las relaciones de género. En sus palabras:

La UE tiene un compromiso explícito con la igualdad de género y la justicia [... y] este compromiso ha recibido una atención merecida, pero los compromisos patriarcales más implícitos permanecen en la institucionalización de la masculinidad hegemónica y en la forma en que la UE organiza su espacio económico. Estos compromisos se hacen visibles en la investigación que analiza la integración europea a través de una lente de género (LOCHER; PRÜGL;2009,p. 95).

\footnotetext{
${ }^{3}$ Esto no implica per se que la integración de conocimientos de redes periféricas sea subordinada; sino que existen márgenes de autonomía que los y las académicas utilizan para ganar reconocimiento, visibilidad y circulación en sus redes locales y regionales; y como puerta par a su reconocimiento global (Perrotta; Alonso, 2020)

${ }^{4}$ Excede los objetivos de este trabajo hacer un análisis del desarrollo de este pensamiento.
} 
Así, entre los avances se encuentran las políticas contra el tráfico, las intervenciones administrativas por la igualdad de género, la promoción de agendas diversas, etc. No obstante, en el campo de la investigación se observa que los y las investigadoras feministas están inmersos en sus propias disciplinas y su investigación se basa en perspectivas disciplinarias; destacándoselas RI, la economía política internacional y la política comparada. Los estudios más recientes sobre el rol de la agencia en redes, promovidos especialmente desdeliteratura más sociológica, está contribuyendo a cerrar la brecha entre los compartimentos estancos disciplinares (LOCHER; PRÜGL;2009, p. 196).

En la investigación desde las TNR, desde la economía política internacional (mayoritariamente) se han concentrado los estudios sobre los impactos de la globalización (MARCHAND; 1994; WAYLEN; 2004) y de los regímenes comerciales sobre mujeres, incluyendo miradas queinterseccionan desigualdades (mujeres-rurales;mujeres-indígenas; mujeres - pobres; incluyendo también miradas sobrelas infancias) (ELIAS; RAI; 2015;2019; HOBSON; SEABROOKE; 2007). Estos estudios se emparentan con los antes citados por Locher y Prügl (2009); quizás a modo de hipótesis tentativa para otro trabajo, desde los estudios de géneros y los feminismos se pueden construir puentes de conocimiento entre enfoques teóricos que han permanecido divididos durante mucho tiempo.

Finalmente, desdela geopolítica crítica también se nutre el estudio desdelos feminismos a la integración regional. En este punto se destacan los trabajos pioneros de Almudena Cabezas para mirar desde un prisma feminista y descolonial a colectivos variados que "hacen integración” y "construyen región" (CABEZAS; 2014; CABEZAS; BROCHNER; 2019; CABEZAS; SERNA; 2013). La autora recupera en sus trabajos a los estudios descoloniales, lo que pasamos a revisitar en el siguiente acápite, de manera de completar este primer mapa de literatura disponible.

\section{Los feminismos descoloniales en diálogo con la construcción de región}

Los enfoques feministas y descoloniales permiten aproximarnos a las diferentes aristas de la integración en el MERCOSUR con nuevas lentes, para comprender de forma más cabal cómo funcionan los procesos de integración en nuestra región, cuáles son sus avances, sus posibilidades y sus desafíos.

El feminismo descolonial latinoamericano (FDL) surge en la década de 1980 como una revisión crítica a los feminismos hegemónicos que intentan establecer una mirada única y universal sobre la mujer, basándose en las preocupaciones y realidades de las mujeres blancas occidentales europeas y norteamericanas. Sin desconocer estos aportes, busca tomar en cuenta todos los elementos que estas autoras no miraron por no formar parte de sus experiencias vitales (VILLARROEL PEÑA; 2018). Así, el FDL abreva en la tradición del pensamiento crítico latinoamericano en sus diferentes facetas (política, geopolítica, social, cultural, económica, del podery del saber), re -elaborando sus aportes a la luz de las diversas vertientes del pensamiento feminista. Entre las corrientes feministas de las que se nutren podemos mencionar a los feminismos negros (COLLINS; 2000; HOOKS; 1989), los feminismos poscoloniales (SPIVAK; 2003; MOHANTY; RUSSO; TORRES; 1991), el feminismo autónomo o los feminismos descoloniales (como los feminismos indígenas o el feminismo comunitario) (VILLARROEL PEÑA; 2018).

Los feminismos negros son los primeros que se posicionan frente a los feminismos occidentales. Parten de reconocer y visibilizar que no hay una sola forma universal de ser mujer. La etiqueta feminismos poscoloniales engloba a todos aquellos que surgen en el período posterior al movimiento descolonial de África y que abogan también por incorporar a otras mujeres no hegemónicas y periféricas al feminismo (negras, lesbianas, no-occidentales, etc.). El feminismo autónomo retoma influencias del liberalismo, las izquierdas y el anarquismo, postulando la libertad frente a los cánones tradicionales y modernos de opresión y se enfoca en la crítica hacia los feminismos institucionalizados, vinculados al Estado, los partidos políticos o las organizaciones (VILLARROEL PEÑA; 2018). Los feminismos descoloniales también parten de la crítica al feminismo institucionalizado y a la cooperación internacional. Su propuesta se orienta a la autoorganización y la autogestión y a visibilizar las diferentes formas de opresión que se intersectan desde el conocimiento situado. Los feminismos indígenas (PAREDES; 2012; VILLARROEL PEÑA; 2018) tienen como eje la cuestión de la validez de 
las cosmovisiones no hegemónicas. El feminismo comunitario (GUZMAN; PAREDES; 2014), por su parte, propone comprender que existen otras formas de feminismo diferentes al occidental, al que ven como un mecanismo de dominación. De esta forma, el FDL busca proponer líneas de pensamientoy de acción que permitan visibilizar las diversas desigualdades que atraviesan a las mujeres de nuestro continente (BIDASECA;2011; CUMES; S. F.; CURIEL; S. F.; ESPINOSAMIÑOSO; 2014; LUGONES; 2008; PAREDES; 2012; SEGATO; 2012).

El pensamiento descolonial recala en la comprensión de la dimensión global y sus vínculos con el ámbito local para denunciar la colonialidad que perdura en nuestros territorios (en sentido amplio, incluyendolos cuerpos). Desde esta perspectiva, indaga en cuestiones que van desde la geopolítica a la dependencia económica y cultural ola injusticia social en la región. Para ello, propone respuestas desdela resistencia que se vinculan con el intento de de -colonizar el podery el saber. A estas consideraciones, el prisma del feminismo añadela desigualdad de géneroy de sexualidad, queintersecta con todas las demás desigualdades (de raza, de etnia, de clase, de edad, de posición geopolítica) colocando a la mujer latinoamericana en una posición de subalternidad aún mayor. Así, recuperando aquella tradición, el FDL va un paso más allá y propone

\footnotetext{
"otros marcos analíticos para la comprensión de las relaciones sociales, mediante una mirada imbricada de las opresiones de raza, sexo, clases, sexualidad y geopolítica, que el capitalismo, el racismo y patriarcalismo origina en las vidas de las mujeres de la periferia en particular, y en las poblaciones de la periferia global en general.” (VILLARROEL PEÑA;2018, p.106)
}

Así, parten de la revalorización del conocimiento situado y horizontal, sin pretensiones universalistas o de verdades incontrastables, que permita dar respuestas más acertadas y acordes a las problemáticas de la propia región ${ }^{5}$.

Para las RI, en general, y el campo de la integración regional, en particular, el principal aporte de los feminismos está vinculado con repensar el concepto de poder y las luchas de poder (ROBERTS; 2017; VÁZQUEZ; 2012), poniendo de relieve las diferentes formas de opresión que se intersectan y "que nos atraviesan dentro del entramado de jerarquías, tanto a los sujetos como a los instrumentos/mecanismos de orden/poder global” (RELACIONES INTERNACIONALES; 2014,p.7). En este marco, el pensamiento FDL aporta reflexiones que apuntan a la crítica al orden internacional y acciones que buscan subvertirlo desde la promoción de la horizontalidad delas relaciones y de la desarticulación de las relaciones estructuradas en torno de la masculinidad. La crítica central se orienta a develarel racismoy otras múltiples desigualdades intersectadas que atraviesa el sistema internacional tal como está construido y funciona en la actualidad. Así, pretende explicar las formas de actuación de aquellos que ejercen y/o disputan el ejercicio de poder.

Partiendo de estos enfoques, se abren nuevas preguntas sobre y para la integración regional latinoamericana: ¿Cuáles son los roles socialmente construidos y asignados a los hombres y mujeres en los procesos de integración en la región? ¿Con que otras desigualdades se intersectan estas relaciones jerárquicas de género? ¿Cómo cristalizan estas relaciones de poder en la construcción de la institucionalidad? ¿Cómo los valores vinculados a esas diferentes jerarquías moldean el proceso de integración?? ¿Cómo impactan en la construcción y contestación de los procesos de integración los afectos, las emociones y las corporalidades? ¿Cómo y dónde se incluyen espacios vinculados a los géneros en estos procesos? ¿Cuáles son las consecuencias de su instalación? ¿Es la introducción de estas voces feministas un des afío a los espacios masculinizados?

Estas preguntas, que retomamos en la sección final para la propuesta de una agenda de investigación sobre el MERCOSUR pero con capacidad de viajar y ser traducida en otras latitudes, surgen de la indagación que venimos realizando sobre algunas agendas y pasamos revista a continuación.

\footnotetext{
${ }^{5}$ Cabe señalar que, si bien surge desde América Latina, este feminismo descolonial no se plantea como universal ni invisibiliza otros Sures.
} 


\section{El MERCOSUR con anteojos feministas}

En este trabajo nos cuestionamos ¿dónde están las mujeres en el MERCOSUR?, desde una mirada que interpele la praxis y la teoría de la integración desde los feminismos, con énfasis en las vertientes descolonialeslatinoamericanas. Esto es, pasar de analizar únicamente el rol de las mujeres y su contabilización en espacios institucionales, para tensionar cómo se tejen relaciones de poder, cómo se intersectan desigualdades y cómo pensar una gramática otra de los estudios de la integración y el regionalismo. En virtud de ello, en esta sección presentamos resultados de investigaciones que las autoras hemos desarrollado en los últimos diez años, para argumentar que el campo de estudios de la integración presenta sesgos machistas, de clase y raza que requieren ser visibilizados y deconstruidos para una aprehensión más cabal de los procesos de construcción de región. Este sesgo no es solo atribuible a la producción de conocimiento académico, sino que también constriñen a las políticas públicas regionales.

El MERCOSUR fue creado en el año 1991 con la firma del Tratado de Asunción entre Argentina, Brasil, Paraguay y Uruguay con el objetivo de crear un mercado común. En estos treinta años, se han sumado como Estados asociados Chile, Bolivia, Perú, Colombia, Ecuador, Venezuela, Guyana y Surinam ${ }^{6}$; desde el punto de vista de integración comercial el proceso constituye una zona de libre comercio que avanzó hacia una unión aduanera caracterizada como imperfecta por varios autores y autoras (BOUZAS; 2001; 2011; BOTTO; TUSSIE; DELICH; 2004) y que hoy se encuentra tensionada por divergencias entre los socios a raíz de la dinámica de relacionamiento externo ${ }^{7}$; pero que ha avanzado en la conformación de políticas públicas regionales en agendas positivas. En este proceso, se han dado espacios institucionalizados para la incorporación de la perspectiva de géneros. La producción de conocimiento y las instancias de movilización de colectivas de mujeres y diversidades ha acompañado el proceso, a la par de tensionado por la incorporación de estas otras voces.

Los estudios que han abordado cuestiones de géneroy problemáticas feministaslo han hecho desde tres grandes nudos temáticos: primeramente, las pioneras en iluminar la relación integración y mercado de trabajo han sido las organizaciones sindicales. De la misma manera que los inicios del proceso de integración del MERCOSUR contaron con la movilización sindical en pos de la inclusión de derechos sociolaborales en las reglas del bloque, han sido las mujeres sindicales las que también incorporaron la mirada de género. Así, desde los diferentes organismos que han apoyado la participación sindical regional, se han desarrollado trabajos que iluminaron estos aspectos (ABRAMO; RANGEL; 2003; FAUSTO; 1999; GODIO; 1997; ULSHOEFER;1997). Avanzado el proceso de integración, se han ido incorporando temáticas referidas a migraciones (KOFMAN;RAGHURAM; 2012), por un lado, y a trata de personas (CASTIGLIONI; NEJAMKIS;2014), por el otro.

Un segundo núcleo de trabajos aborda la creación y puesta en marcha de la Reunión Especializada de la Mujer en el MERCOSUR, así como su vinculación con otros espacios institucionales del bloque en el que se incorpora una mirada de géneros. Vale destacar que la incorporación de esta Reunión se vincula con el activismo de colectivo/as de mujeres y/o feministas en el MERCOSUR, como parte de una acción social transnacional ylos debates del bloque (durante esos años) de fomentar un MERCOSUR inclusivoy solidario, fortaleciendola participación institucionalizada de diferentes colectivos (AZAR; CELIBERTI; ESPINO; 2005; DA SILVA; 2008; FERRETTO; PICASSO; 2018; GÓMEZ CASTAÑEDA; NACLERIO TORRES; 2011).

Un tercer abordaje se centra en una mirada que cruza trabajos desde la deconstrucción yla interseccionalidad al analizar en detalle la participación de organizaciones de mujeres en algunas políticas públicas regionales. En este punto, destacamos los trabajos de Jelin (2000) y de Ferro (2008); pero también aquellos que incorporaron por la vía de seminarios reflexiones epistemológicas y metodológicas parala inclusión de las mujeres y otras diversidades en las agendas de política (ICAZA GARZA; 2007; MERCOSUR \& AECID; 2009). En esta última gramática encontramos los puntos convergentes con los trabajos que realiza Cabezas (antes citada) desde la geopolítica crítica y el feminismo decolonial.

\footnotetext{
${ }^{6}$ Bolivia se encuentra en proceso de adhesión como Estado parte; Venezuela adquirió dicho estatus en el año 2012 y actualmente está suspendido. ${ }^{7}$ Para esto, véase: Costa y Bianco, 2018; Simonoff, 2020.
} 
Sobre esta base aquí no presentamos todos los espacios institucionalizados para la agenda de géneros; sino que mostramos la importancia de incorporar la curiosidad feminista a dos agendas escogidas, como resultado de reflexiones a las que hemos llegado en nuestras investigaciones -y atravesadas por elímpetu delos movimientos feministas - y que abren avenidas de trabajo. Así, la selección de estos dos casos es intencional, fruto de nuestra propia trayectoria, pero que además se justifica porque se trata de una agenda que desde sus inicios incluyó el abordaje de los géneros (mujeres rurale s) y otra que, pese a ser un espaciofeminizado (educación) no se han permeadoen resultados de políticas pese a las instancias de movilización social en cada país.

\section{La agenda de agricultura familiar}

En el año 2004 se instaló en la estructura del MERCOSUR la Reunión Especializada de Agricultura Familiar (REAF) (Res. GMC N¹1/04), con el objetivo de fortalecer las políticas públicas para este sector de la agricultura de la región. La instalación de la REAF resultó una decisión política inédita para la región, en el marco de una disputa de larga data entre diferentes modos de ser y estar en el campo (GRAS; HERNANDEZ;2009). En este proceso se conjugaron cuatro elementos, a saber:1) la demanda de las organizaciones de la agricultura familiar;2) el particular contexto político regional, marcado por el ascenso de nuevos gobiernos en los Estados Parte del MERCOSURy por el pasaje a un nuevo tipo de integración, más solidaria e inclusiva, no tan centrada en la integración comercial y con un nuevo rol para las y los actores sociales (PERROTTA; 2013; VAZQUEZ; 2011); 3) el liderazgo de Brasil en el abordaje de la agricultura familiar; y 4) el acompañamiento (técnico y financiero) de organismos y agencias internacionales sectoriales, en especial el Fondo Internacional de Desarrollo Agrícola (FIDA) (GONZÁLEZ; 2019).

En términos institucionales, la REAF adquirió la forma de Reunión Especializada, un espacio consultivo ideado para dar cauce a tópicos no contemplados en los grupos de trabajo instalados al comienzo del proce so de integración regional, por el énfasis en aspectos comerciales de la estructura decisional primaria. Este nuevoórgano resultó novedoso en dos aspectos: primero, por su agenda y objetivos; segundo, por la dinámica de trabajo establecida (GONZÁLEZ; 2018). En efecto, el espacio que había sido erigido con fines principalmente comerciales (facilitar y promover el intercambio de los productos de la agricultura familiar) incorporó rápidamente nuevas aristas, consolidando en tan sólo dos años una agenda nutrida e integral, vinculada a la concepción de la agricultura familiar no sólo como un sector productivo sino también como un sector social y cultural diferente a otros que habitan el campo. Parte de este proceso fue la incorporación de Grupo de Trabajo (GT) ${ }^{8}$ de Género en el año 2005.

La creación de este GT estuvo vinculada con una realidad que atraviesa el agro, particularmente en nuestra región: la constitución patriarcal de las familias rurales y la asociación de lo campesino con la masculinidad. Desde esta perspectiva no sólo se sostiene una construcción de los órdenes de género que subordina a las mujeres y diversidades que habitan los espacios rurales, sino que, principalmente, se invisibilizan los múltiples roles (domésticos, comunitarios, productivos) de estas actoras y se obstaculiza la posibilidad de reunir información fidedigna y confiable respecto de la situación de las mujeres y otras diversidades en la ruralidad. Así, se impide la puesta en marcha de políticas públicas efectivas para abordar las ruralidades conosureñas, en tanto no se cuenta con una imagen completa de las realidades de los espacios rurales. Además, la ausencia de espacios orientados al abordaje de estas temáticas en las estructuras institucionales de los Estados Parte y Asociados, pero también de espacios de diálogo regional, fue lo que motivó la instalación de este GT. Con ello, la REAF buscó realizar estudios sobre la situación de las mujeres rurales, sistematizar

\footnotetext{
${ }^{8}$ Los GT son espacios dentro de las reuniones especializadas (RE) para atender de forma profunda a los ítems que conforman su agenda de trabajo. Los mismos varían de acuerdo con las necesidades de la RE y su funcionamiento es previo a cada instancia plenaria, ya que allí se llega a los acuerdos básicos sobre los que se avanzará en el desarrollo de la reunión. En la REAF, la consolidación de estos grupos permitió que a su interior se multipliquen las cuestiones abordadas, así como los enfoques y perspectivas, lo que ha dado como resultado una cada vez mayor complejización de las temáticas trabajadas y un tratamiento que aparece como más adecuado y completo.
} 
experiencias de políticas públicas de género en la región y estimular la participación de mujeres en las delegaciones nacionales, tanto gubernamentales como de la sociedad civil.

El abordaje de estas temáticas en el ámbito regional permitió extender el tópico hacia otros sectores del MERCOSUR (por ejemplo, a través de articulaciones con la Reunión Especializada de la Mujer) y constituyó el puntapié para dar mayor relevancia a las cuestiones de género en las arenas nacionales (GONZÁLEZ, 2018).De estaforma se expresa en las entrevistas realizadas:

\begin{abstract}
"la instalación de la temática de género en la agricultura familiar fue un acierto muy importante, con resultados muy interesantes con relación a cómo los funcionarios percibían el tema género y adaptaban políticas para el tema género." (Entrevista a técnico de la delegación argentina)

"Y en un tema que resulta bastante complicado de trabajar, y porque... no porque sea árido... es porque es complicado entender que necesitamos transversalizar la perspectiva de género en políticas públicas, muchas de las cosas que hemos podido avanzar ha sido porque ese tema tiene un posicionamiento muy fuerte desde la REAF. Y permea, por lo menos intentamos acá en Uruguay que permee, hacia el trabajo que hacemos." (Entrevista a técnica de la delegación uruguaya)
\end{abstract}

En términos de las dinámicas de trabajo establecidas, la REAF también resultó novedosa, como se mencionó. En efecto, en este espacio la participación de actores y actoras sociales es distintiva en relación con lo ocurre en el resto del MERCOSUR, ya que fue más amplia y profunda. Es decir, en el ámbito de la REAF, numerosos representantes de las organizaciones de la agricultura familiar de la región participaron incorporando sus propios puntos de vista en las discusiones y añadiendo temas a la agenda de trabajo (GONZÁLEZ; 2020). Pero, además, se e stablecieron dinámicas que favorecieron la participación de mujeres rurales en una arena de acción sumamente masculinizada. Como se señala en una de las entrevistas realizadas,

\footnotetext{
"lo cierto es que cuando se convocaba, siempre venían varones. Los representantes y los dirigentes más fuertes del campo son varones [...] casi el 99,9\%. Porque [las organizaciones de Argentina] tiene muchas mujeres, pero la parte dirigencial la tienen los varones" (Entrevista a representante de organización de agricultura familiar de la delegación argentina).
}

La REAF, sin embargo, adoptó una estrategia novedosa, promovida por la Sección Nacional de Argentina:

\begin{abstract}
"Si venían varones, venía uno sólo. Si no, se le podía dar una segunda oportunidad de que venga otro acompañante en la medida que sea mujer ojoven, esas eran las estrategias. Así empezamos y así empezó a cruzar el tema transversalidad de género" (Entrevista a representante de organización de agricultura de la delegación argentina).
\end{abstract}

De esta forma, se promovió una participación más igualitaria al interior de la REAF, que redundó en que, entre los años 2004 y 2014, participaran 260 mujeres en representación de las organizaciones de agricultura familiar de los Estados Parte, frente a 332 hombres. Inclusola representación de las mujeres en el ámbito de las organizaciones superólo ocurrido con los y las representantes de los Estados, donde en el mismo período participaron 187 mujeres y 339 hombres (GONZÁLEZ; 2020). Asimismo, esta estrategia tuvo un fuerte impacto al interior de las organizaciones -en especial en aquellas que no eran exclusivamente de mujeres rurales - que comenzaron a incorporar perspectivas -y prácticas - de género. De esta forma es señalado en las entrevistas realizadas:

"La estrategia era también convocar varones y mujeres. Entonces la lógica de valor de que en la representación vinieran mujeres, fue para mí de la experiencia más enriquecida para poder construir una heterogeneidad de representación" (Entrevista a representante de organización de agricultura familiar de la delegación argentina).

"[La dinámica de trabajo] puso en cuestionamiento a algunas organizaciones y algunos modos de funcionamiento patriarcales de algunas de las organizaciones de la sociedad civil. O sea que sirvió de 
cuestionamiento para algunas organizaciones.” (Entrevista a investigadora de la delegación de uruguaya)

"Yo he escuchado a los mismos en períodos más iniciales hablar de un modo y después se cuidan mucho lo que dicen. Y también es correcto, porque cuando tienen que tomar la palabra en una mesa donde ya esa cuestión, por lo menos lo políticamente correcto, está muy asimilada, los mismos referentes han cambiado mucho el lenguaje para hablar en términos de género. Y muchos se forman en perspectiva de género.” (Entrevista a investigadora de la delegación argentina)

En la realización de sus actividades, el GT contó con un fuerte apoyo de la Agencia Española de Cooperación Internacional para el Desarrollo (AECID), que contribuyó con el financiamientoy la organización de un amplio número de actividades, en particular durante sus primeros años (MERCOSUR \& AECID; 2009).En cuanto a las acciones encaradas desde este GT, se destaca la aprobación, en el año 2005, de la Recomendación “Bases para una política de género en la agricultura familiar”, que postula directrices vinculadas con la ampliación del acceso de las mujeres a los programas de crédito y comercialización, las garantías de titulación conjunta de la tierra para varones y mujeres o el desarrollo de Programas Nacionales de promoción de la equidad de género en el campo, en particular en lo que respecta a la capacitación y el acceso a derechos.

Como se puede observar hasta aquí, en el caso de la REAF la incorporación de una perspectiva de género contó con el impulso (económico y técnico) de los organismos internacionales, a lo que se sumaron estrategias nacionales (en cierta medida aisladas) que partieron de miradas atentas sobre lase realidades del agro regional. Si bien en un principio estas acciones fueron sencillas y no se plantearon modificar de raíz las desigualdade s de género que permean los mundos rurales, la rápida adopción de estas estrategias por parte de las actoras sociales fue lo que otorgó una nueva dinámica al abordaje de estas cuestiones. Así, desde una primera mirada es posible advertir cómo estas accione s que aparentan sencillas y minúsculas redundan en efectos palpables, tanto en lo que refiere a las agendas como en las dinámicas de acción política de los colectivos agrarios. Estas constataciones, sin embargo, abren lugar a nuevas preguntas sobre las que resulta relevante indagar: ¿se mantuvieron estas acciones a lo largo del tiempo o lograron profundizarse? ¿cómo estas acciones impactaron en el resto de la agenda de la REAF? ¿En qué medida son los géneros -la agenda y las dinámicas-lo que permite explicar al menos ciertos avances de la integración en esta área? ¿Es posible extender esta mirada al resto del MERCOSUR?

\section{Lagenda educativa}

La agenda educativa data del inicio del MERCOSUR ya que la conformación de una comunidad regional precisaba del desarrollo de una conciencia ciudadana favorable a partir de procesos socioeducativos, además de contribuir al objetivo de libre movilidad Así, la configuración de un mercado educativo a nivel internacional que tensionó la provisión pública de este servicio y la creciente tendencia hacia su mercantilización, un aspecto que no fue disonante respecto de la orientación general del bloque en esta fase inicial. Además, la necesidad de crear una identidad regional que legitimara el proceso de integración a partir del fortalecimiento de la ciudadanía. De la convergencia de estos elementos se institucionalizó el Sector Educativo del MERCOSUR (SEM) y se inició un proceso de acumulación de capacidades y experiencias que acompañólos cambios políticos nacionales ylas etapas por las que transitó el MERCOSUR.

El SEM se organiza en cuatro áreas de trabajo: educación básica, educación técnica, educación superior y formación docente; que, a diferencia de otras agendas no comerciales (ya sean reuniones ministeriales o especializadas) desarrollan sus tareas desde un nutrido entramado institucional a partir de planes de trabajo plurianuales. Al igual que la mayoría de las instituciones del MERCOSUR, el SEM funciona por medio de delegaciones nacionales: el trabajo diario se realiza en cada Estado Parte y Asociado por funcionarios/as nacionales que desarrollan las actividades en áreas cooperación internacional o bien las de competencia temática -como puede ser la acreditación de carreras, las políticas socioeducativas o la elaboración de estadísticas-. Esto significa que sus competencias no son exclusivas del MERCOSUR, sino que esta tarea de vinculación internacional-regional se incorporara a un conjunto de atribuciones relativas al cargo 
particular que desempeñan. También implica que el trabajo en conjunto de todas las delegaciones nacionales se realiza en el marco de las reuniones semestrales (en cada Presidencia Pro Tempore, PPT) y las reuniones preparatorias que tengan antes de éstas. Esta modalidad implica que el funcionamiento del Sector depende de las erogaciones de recursos del presupuesto de cada Ministerio, los fondos que cada país debe girar al Fondo Educativo del MERCOSUR (FEM) y los que consiga por medio de la cooperación internacional (comola Organización de Estados Americanos, OEA; Organización de Estados Iberoamericanos, OEI; el Fondo de las Naciones Unidas para la Infancia, Unicef; la Unión Europea, UE; entre otros).

Las metas del SEM se han mantenido -con poca variación-a lo largo del tiempo y desde allí se desprenden los objetivos estratégicos y actividades que han sido plasmados en los planes de trabajo, canalizando a lo largo del tiempo diferentes proyectos de integración. Así, al analizar las finalidades hacia las que se orienta el ejercicio del poder político regional por medio de la puesta en marcha de políticas públicas regionales, se observa cierta hibridez del MERCOSUR (PEIXOTO; PERROTTA; 2017): desde aquellas que acompañan sin tensionar el modelo de regionalismo abierto -basado en la centralidad de la liberalización comercial-hasta las que se corresponden con los lineamientos del regionalismo "post" (posliberal, post-hegemónico)-caracterizado por la recuperación de la política y del rol de conducción de los Estados (por sobre el mercado) de la integración, la incorporación de agendas positivas de integración y la preocupación por los aspectos de participación social y ciudadana, la reducción de asimetrías y la persecución de metas autonómicasy desarrollistas-.

Esta versatilidad explica el desarrollo de la agenda de integración educativa a lo largo del tiempo, especialmente desde la posibilidad de su creación en el contexto de un proyecto regional adverso a la integración social, pasado por la gravitación de la educación en el marco del regionalismo post yla actual resiliencia en una coyuntura prima facie adversa. Así, el desarrollo de las políticas puede analizarse a partir de cuatro etapas, a saber:1) construcción de confianza (19912001);2) visibilización de la agenda educativa como parte del giro hacia un Mercosur social, solidario y ciudadano (20022011);3) ampliación de la agenda (2012-2015); 4) resiliencia (desde el 2016) (para cada una, PERROTTA;2019). A pesar de este prolífico desarrollo, en treinta años de integración educativa las cuestiones de géneros no formaron parte de la agenda institucional formal del SEM, pese a que las cuatroáreas de trabajo son susceptibles de ser abordadas desde ese prisma.

La problematización en torno a los géneros y las propuestas desde los feminismos surge en la agenda de participación y movilización social: puntualmente, en el marco de las iniciativas de participación de los y las jóvenes de escuela media en el Parlamento Juvenil del MERCOSUR (PJM); y como reclamo de parte de las organizaciones sindicales que no tienen canalización formal de sus instancias de participación en el SEM. De manera más tangencial, en la agenda formalizada, algunas cuestiones fueron permeadas de manera reciente (e inconclusa a raíz de la pandemia) en la comisión ad hoc de educación inicial a partir del trabajo con Unicef para la realización un seminario que mapee las prácticas nacionales en este nivel educativo: las políticas para la primera infancia son nodales en la discusión de las políticas de cuidado. Por lo tanto, conforme avance la negociación regional en esta área, se podrían empezar a generar espacios de discusión proclives a incorporar nuevas miradas, menos masculinizadas y conservadoras del statu quo regional. Sin embargo, aquí, es menester destacar, que esta incorporación está traccionada por la articulación con el espacio institucional del MERCOSUR dedicado al desarrollo social y el trabajo con un cooperante como Unicef.

El PJM fue lanzado en el año 2010y tiene como objetivo generar espacios de participación para que los ylas jóvenes intercambien, dialoguen y discutan entre ellos/as problemáticas fuertemente vinculadas con sus vidas, presentesy futuras. Así, se genera un espacio de diálogo para, primero en sus países y luego con sus pares regionales, compartan sus visiones e ideas acerca de qué escuela media quieren y se difundan valores democráticos comola participación ciudadana, el respeto por la democracia, los Derechos Humanos, la conciencia social, la cultura de la paz y el medio ambiente. Las primeras ediciones contaron con fondos de la OEA y se viene realizando con una frecuencia bianual (salvo en el 2021 que fue puesto en suspenso a raíz de la pandemia, además de otras dificultades del SEM como parte del proceso de recambio gubernamental dela mayoría delos países). En todas las declaracioneslos y las jóvenes incorporan la dimensión de géneros como un eje de trabajo; sibien las propuestas de políticas y acciones han seguido una lógica incremental (que incluye desde 
promoción de educación sexual integral, hasta registros que eviten la fijación sexo genérica y baños mixtos como política educativa). La última edición se dio en el marco de un proceso de tensión con la delegación de Brasil, que llevó a que ese país dejara de participar del SEM (situación inédita):

"la visión de Brasil era que los jóvenes no participen, especialmente para que estos temas [los de género, ambiente] saltaran y generaran conflictos con su política interna... por lo tanto, todo el proceso previo a la realización del PJM fue complicado... el hecho de que no envíen delegación y que encima nos declaren a los demás en rebeldía muestra cuán difícil está siendo conjugar la agenda de los jóvenes con la agenda regional" (entrevista a funcionario de la delegación uruguaya).

"en este MERCOSUR no se pueden incorporar cuestiones de género si queremos seguir avanzando en los demás proyectos regionales. Paraguay plantea resistencia; del nuevo gobierno de Uruguay nolo tenemos aún muy claro; y de Brasil esperamos que vuelva, entonces poner un tema que ya sabemos que es conflictivo no es estratégico" (entrevista a funcionaria de la delegación argentina).

"nosotres en la edición nacional decidimos que iban a estar todos los temas propuestos por les pibis; los feminismos y los movimientos ambientales están muy fuertes en la agenda actual y queremos potenciar su voz porque el PJM es una herramienta de participación política... por eso entendemos que diferenciamos la edición nacional de la regional, donde intentamos colocarlo, pero fue vetado por Paraguay... por eso, el nuevo plan de trabajo del Sector no menciona estos nuevos temas... esperamos que vengan mejores tiempos para si incluirlos de lleno" (entrevista a funcionario de la delegación argentina).

Con todo, el ímpetu transformador de la agenda de integración educativa regional que plantea la política del PJM sirvió para la movilización nacional y regional de jóvenes, y esa acumulación de capacidades y de voces choca con el momento actual que está experimentando el SEM.

Finalmente, la participación de sindicatos docentes en el SEM no está formalizada y depende de las diferentes gestiones que se hagan en cada PPT. Aun cuando participen, lo hacen con voz y sin voto, ni con posibilidades de proponer proyectos regionales. Sibien en otros momentos del proceso de integración (especial mentela etapa 2002-2015) contaron con capacidad de incidencia y de presionar por cierto accountability de las estructuras formales, en la fase de resiliencia del SEM esto se fue perdiendo. En entrevista con un referente sindical de Argentina, se indicó cómo fueron perdiendo posibilidades de participación y cómo esto redunda en acciones menos sustantivas:

\footnotetext{
"si nos hubieran convocado, todas las estrategias transnacionales que llevamos adelante en pandemia podrían haber nutrido al sector de temas ausentes; esto incluye desde lo específico del trabajo docente con nuevas herramientas y plataformas, pero también cómo se abordan los cuidados y las violencias”
}

Así, y a la luz de la experiencia de la REAF, se comprueba la importancia de los movimientos sociales y de la organización de la participación (en este caso, de los y las jóvenes) para el avance de estas agendas que buscan reducir desigualdades. En la medida en que el SEM sigue siendo un espacio institucional que no permite la inclusión de voces no gubernamentales, la inclusión de agendas “nuevas” será a destiempo de los avances políticos y normativos nacionales, muchas veces fruto de movimientos de contestación y reclamación populares.

\section{Conclusión}

La integración regional, como hemos aprendidoy reproducido desde las teorías, no es un fin en sí mismoni está dada por una esencialidad geográfica. La integración regional es una política pública que se orienta al ejercicio del poder político y las regiones son construcciones sociales políticamente contestadas. En esos proyectos en pugna y contestados, las luchas de los feminismos han estado obturadas. Incluso, aun cuando diferentes esquemas regionales (incluyendo el MERCOSUR) han avanzado en la promoción de regímenes de género y la agenda de investigación ha ido incorporando, paulatinamente, estudios desde los géneros y los feminismos, existen límites implícitos colocados por prácticas masculinizadas y la preeminencia de lógicas patriarcales. Si la integración regional ha de contribuir a la mejora de las 
condiciones de vida de sociedades tan desiguales comolas nuestras, entonces, la integración ha de ser feminista. Además, nuevas formas de construcción de la unidad, tanto como estrategia política como narrativa integracionista que no oprima diferencias, han de nutrirse en buena medida de las prácticas de diferentes colectivos y colectivas en clave feministas y diversos.

Como investigadoras de este campo de estudios y con un anclaje disciplinar a veces en diálogoy a veces en tensión con las RI, y atravesadas por la fuerza de los movimientos de mujeres que están impactando en la política y las políticas en toda agenda y nivel (local, nacional, regional, global), nos cuestionamos nuestra propia práctica académica a la vez que interrogamos y generamos nuevas prácticas en nuestra propia reproducción de la vida cotidiana. Así, comprometernos a dar voz a quien no tienen voz validada es un ida yvuelta, porque nos incluye también a nosotras comoinvestigadoras.

Sobre este marco de relaciones de fuerzas, construcción de poder (tanto en la integración como en la academia) el objetivo de este trabajo consistió en visibilizar las ausencias y mostrar la riqueza del estudio desde primas feministas para establecer una agenda de investigación integral de la integración regional en el MERCOSUR y que recupere el rol de las colectivas de mujeres y diversidades en la construcción de región a partir de su movilización, participación y reclamo. No obstante, recorrer esta vinculación no implica relatar ni contabilizar -en las agendas de políticas regionales, en las currículas académicas y en las citaciones de artículos - dónde están las mujeres desde una perspectiva de la experiencia, sino ir más allá para plantear cómo estos enfoques nos pueden ayudar a comprender teóricamente los procesos que buscamos explicar.

Por este motivo, partiendo de la incorporación de los feminismos en una disciplina androcéntrica (y occidocéntrica) que está en proceso de deconstrucción, es posible cuestionar un campo de estudios que abreva de múltiples disciplinas pero donde hay triples jerarquías que lo configuran -la jerarquía de las RI por otras disciplinas, la jerarquía de la colonialidad del saber (que afecta la producción de conocimiento desde “el Sur”) y la jerarquía patriarcal por nuestra condición sexo genérica. Sobre estos otras y otros que generaron conocimiento antes que nosotras y que están (re)produciendo en múltiples latitudes y cuestionando los regímenes de género, pero también de verdad y saber, recuperamos y partimos de la validez de identificar los espacios institucionales habilitados a la participación de la(s) mujer(es) en el MERCOSUR. Estamos conscientes de su necesaria visibilización para mostrar techos de cristal y pisos pegajosos, jerarquías y desigualdades en cada agenda institucional y en la transversalización de la problemática. No obstante, también nos mueve ir más allá: buscar cómo se cruzan e intersectan diferentes y variadas desigualdades, que involucran no sólo al género sino también a la raza, la clase, la sexualidad y la posición geop olítica, entre otras dimensiones posibles. Porque esa gramática Otra, inclusiva, ha de incorporar a todas, todos y todes (permita ellector aludir por única vez una categoría no binaria y más inclusiva, aunque difícil de procesar en la narrativa académica).

Así, una agenda de investigación feminista decolonial del MERCOSUR no se agota en la necesaria visibilización de las múltiples desigualdades, subalternidades y jerarquías que atraviesan a los y las sujetas que construyen y contestan estos espacios políticos ni en el relato sobre las estrategias de disputa en marcha. Analizar la integración regional desde una perspectiva feminista y decolonial no implica quedarse en una perspectiva de la experiencia, sino ir más allá, para plantear cómo estas experiencias y estos enfoques habilitan preguntas (y respuestas) que nos pueden ayudar a comprender teóricamente los procesos que buscamos explicar. Desde allí, el esfuerzo necesario resulta en la formulación de nuevos interrogantes que interpelen a los procesos de integración desde aristas no contempladas, que han quedado en los márgenes en tanto no son visibles para aquellos y aquellas que piensan, hablan y actúan desde otras historias y otras realidades peroque se develan centrales para comprender y explicar los procesos de nuestra región.

La literatura y la movilización desde los feminismos, a nuestro entender, es vital para generar puentes y cerrar brechas. Es decir, articular corpus de conocimiento y luchas sociales que han permanecido segmentadas por la lógica patriarcal del poder; mientras se construye poder popular para eliminar diferentes desigualdades. Desdela experiencia de 
los FDL podemos generar estos vasos comunicantes para analizar más cabalmente cómo se construye región, cómo se contesta e interpela esa región y cómo la movilización social de reclamo configura un espacio más habitable.

\section{Referências}

ABRAMO, Laís; RANGEL, Marta (2003). Negociación colectiva y equidad de género en el contexto del MERCOSUR. En E. De la Garza Toledo y C. Salas (Eds.), Nafta y Mercosur: Procesos de apertura económica y trabajo (pp. 215-242). Buenos Aires: CLACSO.

ACHARYA, Amitav. (2014). Global International Relations (IR) and Regional Worlds A New Agenda for International Studies. International Studies Quarterly, 58(4), 647-659.

ACHARYA, Amitav; BUZAN, Barry (2019). The Making of Global International Relations: Origins and Evolution of IR at its Centenary. Cambridge: Cambridge University Press.

AZAR, Paola; CELIBERTI, Lilián; ESPINO, Alma (2005). La perspectiva de género en el marco de la integración regional en el MERCOSUR: hacia la construcción de una institucionalidad equitativa. Documento preparado para la COMISEC. Montevideo: COMISEC.

BIDASECA, Karina (2011). "Mujeres blancas buscando salvar a mujeres color café”: desigualdad, colonialismo jurídico y feminismo postcolonial. Andamios, 8(17), 61-89.

BIDASECA, Karina (2018) La revolución será feminista o no será. La piel del feminismo descolonial. Buenos Aires:2018.

BOURDIEU, P. (2003). El oficio de científico. Ciencia de la ciencia y reflexividad. Barcelona: Anagrama.

BOURDIEU, P. (2009). Intelectuales, política y poder. Buenos Aires: Eudeba.

BOUZAS, Roberto (2001). El MERCOSUR diez años después. ¿Proceso de aprendizaje o deja vu? Desarrollo Económico, 41(162), 179 200.

BOUZAS, Roberto (2011). Apuntes sobre el estado de la integración regional en América Latina. En G. Caetano (Ed.), MERCOSUR 20 años (pp. 75-88). Montevideo: Trilce-Cefir.

CABEZAS, Almudena; SERNA, David B. (2013). Cuerpos, espacios y violencias en los regímenes biopolíticos de la Modernidad. De maricas y homosexuales habitando "lo femenino". Política y sociedad, 50(3), 771-802.

CABEZAS, Almudena (2014). Transnational Feminist Networks Building Regions in Latin America. Latin American Policy, 5(2), $207-$ 220.

CABEZAS, Almudena; BROCHNER, Gabriela P. M. (2019). The new cycle of women's mobilizations between Latin America and Europe: a feminist geopolitical perspective on interregionalism Critical geopolitics and regional (re) configurations (pp. 178-196): Routledge.

CASTIGLIONI, Celeste; NEJAMKIS, Lucila (2014). Reflexiones y lecturas en torno a la trata de personas en el Mercosur y la Argentina. Politeia, 37(53), 143-167.

COLLINS, Patricia Hill (2000). Black feminist thought: Knowledge, consciousness, and the politics of empowerment. New York: Routledge.

COSTA, Augusto; BIANCO, Carlos (2018). El Mercosur y su trilema. BORDES(9), 193-204.

CUMES, Aura (s. f.). Conferencia presentada en 1er Coloquio Internacional sobre Transculturalidad Pensamiento y Estética Descoloniales. GLEFAS/UNAM, 8.

CURIEL, Ochy (s. f.). Conferencia Feminismo decolonial latinoamericano y caribeño. Aportes para las prácticas políticas transformadoras. CICODE, Universidad de Granada.

DA SILVA, Roberta (2008). Género e Integración Regional: luces y sombras de la Reunión Especializada de la Mujer en el MERCOSUR. Universidad del Salvador, Buenos Aires.

ELIAS,Juanita; RAI, Shirin (2015). The everyday gendered political economy of violence. Politics \& Gender, 11(2), 424-429.

ELIAS, Juanita; RAI, Shirin (2019). Feminist everyday political economy: Space, time, and violence. Review of International Studies, 45(2), 201-220.

ENLOE, Cynthia (2014). Bananas, beaches and bases: Making feminist sense of international politics. Univ of California Press. ESPINOSA-MIÑOSO, Yuderkys (2014). Una crítica descolonial a la epistemología feminista crítica. El cotidiano, (184), 7-12.

FAUSTO, Ayrton. (1999). Mulher e Mercosul. Mujer y Mercosur. Fortaleza: FLACSO/BID/UNIFEM/UNESCO. 
FERRETTO, Martina; PICASSO, Yamila. (2018). La agenda de género en el MERCOSUR: logros y desafíos, ¿qué ocurre con los derechos sexuales y reproductivos? Revista Electrónica Instituto de Investigaciones Jurídicas y Sociales AL Gioja (20), 35-64.

FERRO, Silvia Lilian (2008). Género y propiedad rural. Buenos Aires: Secretaría de Agricultura, Ganadería, Pesca y Alimentos.

FONSECA, Melody (2020). Global IR and Western Dominance: Moving Forward or Eurocentric Entrapment? Millennium, 0(0), 0305829819872817.

GODIO,Julio (1997). El Mercosur: evolución institucional y papel de las organizaciones sindicales. En OIT (Ed.), El Mercado Común del Sur: MERCOSUR. Estudio de caso sobre una experiencia de integración económica (pp. 28-43). Ginebra: OIT.

GÓMEZ CASTAÑEDA, Eugenia; NACLERIO TORRES, Luiz (2011). "Mujer y MERCOSUR": una mirada retrospectiva y analítica sobre la incorporación de la agenda de género al proceso de integración regional. Temas de Mujeres, 7(7), 163-189.

GONZÁLEZ, Leticia. (2018) Acción social colectiva y procesos de integración regional en Sudamérica: la participación de las organizaciones de la agricultura familiar en la construcción y el devenir de la Reunión Especializada de Agricultura Familiar del MERCOSUR (2004-2012). Tesis. Maestría en Estudios Latinoamericanos, Universidad Nacional de San Martín.

GONZÁLEZ, Leticia (2019) Cambios y continuidades para la agricultura familiar de la región - REAF 2017. En Vazquez, Mariana (comp.). El MERCOSUR. Una geografía en disputa (pp. 345-366). Buenos Aires: Ciccus.

GONZÁLEZ, Leticia (2020) “Del predio a la región: la participación de las organizaciones de la agricultura familiar en la REA F” Revista Brasileira de Planejamiento e Desenvolvimento, v.9, n.5, pp 799-840

GRAS, Carla; HERNANDEZ, Valeria (2009) La Argentina Rural. Buenos Aires: Biblos.

GROSFOGUEL, Ramón; MIGNOLO, Walter (2008) “Intervenciones descoloniales: una breve introducción”. En Tabula Rasa, N9, pp $29-37$

GUZMÁN, Adriana; PAREDES, Julieta (2014) Entrevista sobre Feminismo Comunal en Chiapas, abril 2014. Disponible en: https://www.youtube.com/watch?v=C612BnFCsyk\&t=1212s

HOBSON, John M.; SEABROOKE, Leonard (2007). Everyday politics of the world economy. Cambridge University Press.

HOOKS, Bell (1989). Talking back: Thinking feminist, thinking black. Boston: South End Press.

ICAZA GARZA, Rosalba (2007). El Movimiento pro-Regionalismos Alternativos frente a la Nueva Relación Estratégica América Latina, Caribe - Unión Europea. Reflexiones desde una perspectiva de género. Mimeo

JELIN, Elizabeth (2000). Ciudadanía, movimientos sociales y Mercosur. Buenos Aires: IDES.

KOFMAN, Eleonore; RAGHURAM, Parvati. (2012). Women, migration, and care: Explorations of diversity and dynamism in the Global South. Social Politics, 19(3), 408-432.

KORNBLIT, Ana Lía (2004). Metodologías Cualitativas en Ciencias Sociales. Modelos y procedimientos de análisis. Buenos Aires: Editorial Biblos.

KREIMER, Pablo (2006). ¿Dependientes o integrados?: La ciencia latinoamericana y la nueva división internacional del trabajo. Nómadas(24), 199-212.

KRONSELL, Annica (2005). Gender, power and European integration theory. Journal of European Public Policy, 12(6), $1022-1040$.

LOCHER, Brigit; PRÜGL, Elisabeth (2009). Gender perspectives. En A.Wienery T. Diez (Eds.), European Integration Theory (pp. 181198). Oxford: Oxford University Press.

LOMBARDO, Emanuela; KANTOLA, Johanna. (2019). European Integration and Disintegration: Feminist Perspectives on Inequalities and Social Justice.JCMS: Journal of Common Market Studies, 57(S1), 62-76.

LUGONES, María (2008). Colonialidad y género. Tabula rasa, (9), 73-101.

MARCHAND, Marianne H. (1994). Gender and new regionalism in Latin America: Inclusion/exclusion. Third World Quarterly, 15(1), 63-76

MERCOSUR, \& AECID. (2009). Igualdad de género en la agricultura familiar del MERCOSUR. Montevideo: MERCOSUR-AECID. MOHANTY, Chandra T., RUSSO, Ann, \& TORRES, Lourdes (1991). Third world women and the politics of feminism (Vol. 632). Indiana University Press.

PAIKIN, Damián; PERROTTA, Daniela; PORCELLI, Emanuel. (2016). Pensamiento Latinoamericano para la integración. Crítica y Emancipación, Año VIII(15), 49-80. 
PAREDES, Julieta (2012). Las trampas del patriarcado. En P. Montes (Ed.), Pensando los feminismos en Bolivia (pp. 89-112). Conexión fondos de emancipación.

PARLAMENTOJUVENIL DEL MERCOSUR (2019) Declaración del PJM, Montevideo. Disponible en:

https://www.educ.ar/recursos/156443/parlamento-juvenil-del-mercosur/download/inline Cosultado: mayo 2021

PEIXOTO,Juliana; PERROTTA, Daniela (2017). El Mercosur en el nuevo escenario político regional: más allá de la coyuntura. Desafíos, $30(1), 91-134$.

PERROTTA, Daniela (2018). El campo de estudios de la integración regional y su aporte a la disciplina de las Relaciones Internacionales: una mirada desde América Latina. Relaciones Internacionales (38), 9-39.

PERROTTA, Daniela (2019). La integración educativa en el MERCOSUR. En M. Vazquez (Ed.), E1 MERCOSUR. Una geografía en disputa (pp. 299-266). Buenos Aires: Ciccus.

PERROTTA, Daniela; ALONSO, Mauro (2020). Cross-National Research Partnerships in International Relations: A Study of Research Groups' Practices of MERCOSUR-Re-Envisioning Scholarly Activities Beyond the Global North-Global South Divide.Journal of Studies in International Education, 24(1), 79-96.

RELACIONES INTERNACIONALES (2014). Feminismos en las Relaciones Internacionales, 30 años después. Relaciones Internacionales.

ROBERTS, Adrienne (2017). The Future of Feminist International Relations. Dyvik, SL, Selby,J. and Wilkinson, R. What's the Point of International Relations 231-241.

SEGATO, Rita Laura (2012). Gênero e colonialidade: em busca de chaves de leitura e de um vocabulário estratégico descolonial. Ecadernos ces, (18).

SIMONOFF, Alejandro (2020). Acuerdos Mercosur-Unión Europea desde la perspectiva de la política exterior Argentina. Íconos. Revista de Ciencias Sociales(68), 57-73.

SPIVAK, Gayarti (2003). ¿Puede hablar el subalterno? Revista colombiana de antropología, 39, $297-364$

STAKE, Roberts (1995). Investigación con estudios de caso. Madrid: Ediciones Morata.

TUSSIE, Diana, BOTTO, Mercedes; DELICH, Valentina (2004).El MERCOSUR en el nuevo escenario político regional. Nueva Sociedad(186), 114-127.

ULSHOEFER, Petra (1997). El Mercosur y los desafíos para la integración y participación de la mujer en el mundo del trabajo. En OIT (Ed.), El Mercado Común del Sur: MERCOSUR. Estudio de caso sobre una experiencia de integración económica (pp. 43-53). Ginebra: OIT.

VÁZQUEZ, Alberto (2012). El Feminismo en la teoría de Relaciones Internacionales: un breve repaso. Revista de Relaciones Internacionales de la UNAM, (114), 143-152.

VILLARROEL PEÑA, Yetzy U. (2018). Feminismos descoloniales latinoamericanos: geopolítica, resistencia y Relaciones Internacionales. Latin American Descolonial Feminisms: Geopolitics, Resistance and International Relations. Relaciones Internacionales, (39).

WAYLEN, Georgina (2004). Putting Governance into the Gendered Political Economy of Globalization. International Feminist Journal of Politics, 6(4), 557-578.

\begin{tabular}{ll} 
Funciones de colaboración ejercidas & \\
\hline Leticia González: & Concepción; Metodología; Investigación; Redacción (borrador original); Redacción (revisión y edición); \\
Daniela Vanesa Perrotta & Concepción; Metodología; Investigación; Redacción (borrador original); Redacción (revisióny edición);
\end{tabular}

